\title{
Immediate removal of indwelling catheters after hysterectomy was not associated with adverse outcomes
}

Dunn TS, Shlay J, Forshner D. Are in-dwelling catheters necessary for 24 hours after hysterectomy? Am J Obstet Gynecol 2003;189:435-7. Does immediate removal of an indwelling catheter after hysterectomy reduce recatheterisations, complications, and
pain more than catheter removal on the first day after surgery?

\section{METHODS}

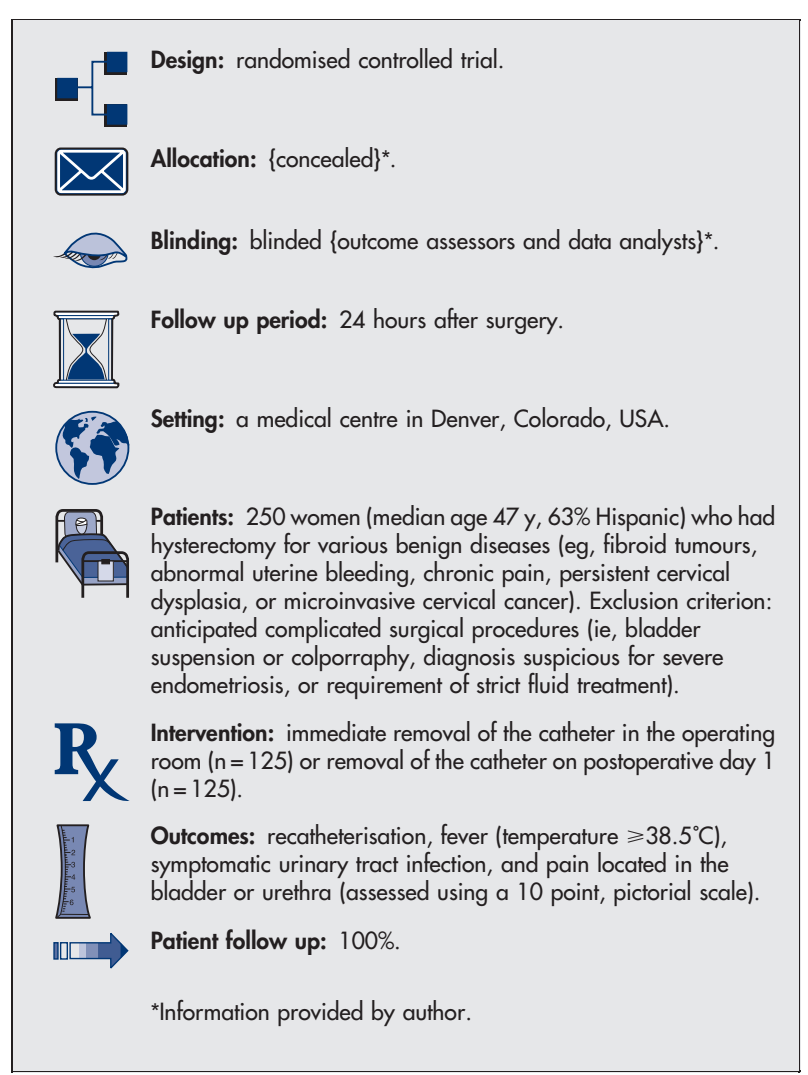

\section{MAIN RESULTS}

Women who had immediate catheter removal in the operating room did not differ from women who had catheter removal on postoperative day $\mathrm{l}$ for recatheterisation for an additional 24 hours $(4.8 \%$ $\left.v 2.4 \%,\{\mathrm{p}=0.31\}^{*}\right)$, fever $\left(4 \% \vee 4.8 \%,\{\mathrm{p}=0.76\}^{*}\right)$, or urinary tract infections $(2.4 \% v 2.4 \%)$. Fewer women in the immediate removal group reported severe pain (table).

For correspondence: Dr T S Dunn, University of Colorado Health Science Center, Denver Health Medical Center, Denver, CO, USA. tdunn@dhha.org Source of funding: no external funding.

\section{CONCLUSION}

Immediate removal of an indwelling catheter after hysterectomy did not differ from catheter removal on postoperative day 1 for recatheterisation, fever, or urinary tract infections, but was associated with fewer reports of severe postoperative pain.

${ }^{*}$ Calculated from data in article.

\section{Commentary}

ndwelling urinary catheters are frequently used after urogenital surgery to monitor urine output and to prevent urinary retention. Although there seems to be no clinical uncertainty about the need for an empty bladder before surgery, controversy arises about the ideal time to remove the catheter. Removal of indwelling catheters tends to be based on personal preference and tradition rather than evidence from research. ${ }^{1}$

The study by Dunn et al was carefully designed, with appropriate and clinically relevant outcomes. The major findings were that patients who had immediate removal of indwelling catheters had no adverse outcomes and reported significantly less pain than patients who had their catheters removed after 24 hours.

These findings are particularly relevant to health professionals in gynaecology, but should also raise questions for health professionals working in other surgical services. Urinary catheterisation is not a harmless procedure. Urinary tract infections account for about $40 \%$ of hospital acquired infections, and $80 \%$ of urinary tract infections acquired in hospital are associated with urinary catheters. ${ }^{2}$ Such infections not only prolong hospital stay and are expensive to treat but also cause unpleasant symptoms. ${ }^{3}$ Health professionals are guided in practice by the ethical principle of "doing no harm." The findings of the study by Dunn et al will support practitioners who want to protect and work in partnership with their patients. Immediate postoperative removal of catheters is likely to restore dignity and control to patients. If catheterisation cannot be avoided, nurses must aim to remove the catheter as soon as possible to alleviate unnecessary pain and other risks to patient safety.

\section{Emil Schmidt, RN, BN (Hon), ICU Reg \\ Dunedin Hospital \\ Dunedin, New Zealand}

1 Fernandez R, Griffiths R, Murie P. Comparison of late night and early removal of short-term urethral catheters. JBI Reports 2003;1:1-16.

2 Stamm WE. Urinary tract infections. In: Bennett JV, Brachman PS, editors. Hospital infections. Fourth edition. Philadelphia: Lippincott-Raven, 1998:477-85.

3 Niël-Weise BS, van den Broek PJ. Urinary catheter policies for short-term management of voiding in hospitalised adults [Protocol]. Cochrane Database Syst Rev 2004;(1):CD004203.

Immediate catheter removal after hysterectomy $v$ removal on postoperative day 1 *

\begin{tabular}{lllll}
\hline Outcome & Immediate removal & Removal on postoperative day 1 & RRR (95\% CI) & NNT (CI) \\
\hline Severe pain $\dagger$ & 0 & $48 \%$ & $100 \%(94$ to 100$)$ & 2 (2 to 3$)$ \\
\hline
\end{tabular}

*Abbreviations defined in glossary; RRR, NNT, and Cl calculated from data in article.

tSevere pain in the area of the bladder or urethra, assessed on a 10 point, self report, pictorial scale. 